\title{
A construção de um litofone em uma abordagem interdisciplinar
}

\author{
Arlino Camargo Livério Jr. \\ Escola de Artes, Ciências e Humanidades, EACH, Univ. São Paulo USP, São Paulo. \\ Eduardo M. Santos \\ Escola de Artes, Ciências e Humanidades, EACH, Univ. São Paulo USP, São Paulo. \\ Adriana P. B. Tufaile \\ Escola de Artes, Ciências e Humanidades, EACH, Univ. São Paulo USP, São Paulo. \\ Alberto Tufaile \\ Escola de Artes, Ciências e Humanidades, EACH, Univ. São Paulo USP, São Paulo. \\ Carlos Molina Mendes \\ Escola de Artes, Ciências e Humanidades, EACH, Univ. São Paulo USP, São Paulo. \\ Rosely Aparecida Liguori Imbernon \\ Programa de Pós-graduação em Ensino e História das Ciências da Terra, IG/Unicamp. \\ Escola de Artes, Ciências e Humanidades, EACH, Univ. São Paulo USP, São Paulo. \\ imbernon@usp.br
}

\begin{abstract}
THE CONSTRUCTION OF A LITOPHONE IN AN INTERDISCIPLINARY APPROACH. Rocks are used in musical instruments since the emerging of music. Lithophones, from the Greek lithos (stone), are percussion instruments based on rock plates. They are similar to the more usual xylophones, but with unique musical characteristics which reflect their peculiar design. In England, the history of lithophones dates back to $18^{\text {th }}$ century. We have constructed and analyzed a lithophone in order to use it as education tool in the teaching of Geology and Physics. An appealing subject, Music, is the central theme in the interdisciplinary approach introduced here. For instance, in the design of the instrument one should characterize the rock plates and know the relation of the frequency and the length of the plates, in order to select particular musical notes. Another goal of the present work is to use the sound of the rock plates as a tool in the characterization of elastic characteristic of materials. Our results suggest that the approach presented should work as an education tool for a broad audience. Citation: Livério Jr. A.C., Santos E.M., Tufaile A.P.B., Tufaile A., Mendes C.M., Imbernon R.A.L. 2014. A construção de um litofone em uma abordagem interdisciplinar. Terræ Didatica, 10(3):283-288. http://www.ige.unicamp.br/terraedidatica/.
\end{abstract}

KEYWORDS: Litophone, geology teaching, interdisciplinarity, elastic proprierties, music.

RESUMO Rochas são usadas como instrumentos musicais desde o surgimento da música. Litofones, do grego lithos (rocha), são instrumentos de percussão baseados em placas rochosas. Eles são similares aos mais usuais xilofones, mas com as características musicais únicas originadas de sua construção peculiar. Na Inglaterra, a história dos litofones data do século 18. Nós construímos e analisamos um litofone com o objetivo de usá-lo como ferramenta educacional no ensino de geologia e física. Um tópico organizador de interesse, a música, é o tema central na abordagem interdisciplinar introduzida aqui. De fato, no desenho do instrumento, as placas de rocha devem ser caracterizadas, com as suas relações entre frequência e comprimento estabelecidas, a fim de que notas específicas sejam selecionadas. Outro objetivo do presente trabalho é a utilização do som como ferramenta na caracterização das propriedades elásticas de materiais. Nossos resultados sugerem que a abordagem apresentada é efetiva como ferramenta educacional para uma audiência ampla.

PALAVRAS CHAVE: litofone, ensino de geologia, interdisciplinaridade, propriedades elásticas, música. 


\section{Introdução}

A importância do som e da sua organização abstrata no desenvolvimento humano é um fato científico ao qual pouco nos atemos. De fato, o som é uma das grandes forças na natureza. Muito antes da utilização de materiais de origem vegetal e animal na produção de instrumentos que reproduzissem os sons da natureza, o homem deve ter utilizado as rochas encontradas no ambiente para esse fim.

Provavelmente, nossos ancestrais perceberam que o som produzido quando se batiam fragmentos de rochas umas contra as outras apresentavam diferentes tipos de sons e tons, em função do tamanho desses fragmentos, e dos diferentes tipos de rochas utilizadas na sua confecção. Essa, talvez, tenha sido a primeira sensação de um ser humano no sentido de produzir um som e controlar ritmo, tonalidade e frequência, embora tais elementos que componham o som ainda fossem somente percebidos pelo sentido da audição.

As rochas são utilizadas em instrumentos de percussão há muitos séculos, e na Inglaterra os litofones, instrumentos de percussão feitos com placas de rochas (hornfels) datam do século XVIII (Bastos, 2010). A palavra litofone é de origem grega (lithos = pedra), e trata-se de um instrumento de percussão, para o qual, em geral, os fragmentos de um mesmo litotipo são cortados em diferentes tamanhos de maneira a produzir melodia e harmonia.

Em documentário exibido pela BBC-Londres, intitulado "Lost Kingdoms of Africa", os diretores apresentam vários exemplos de instrumentos que utilizam rochas e que foram encontrados em diferentes partes do mundo.

A utilização das rochas como material de construção e revestimento ocorre há muitos séculos; em diferentes partes do mundo, assoalhos, colunas, abóbadas, entre outros elementos estruturais, utilizam diferentes tipos de rochas.

Atualmente, o Brasil está entre os cinco maiores produtores mundiais de rochas ornamentais e gera mais de 100 mil empregos diretos nesse setor; a utilização de rochas na construção civil envolve grande variedade de litotipos, desde rochas magmáticas (granitos, sienitos), rochas metamórficas (ardósia, mármores) e mesmo rochas sedimentares (varvito), porém a origem/gênese das rochas, suas características e diferenças são completamente desconhecidas pela grande maioria da população.

Em termos dos litotipos utilizados pelo setor de rochas ornamentais, a escolha de uma rocha como "rocha ornamental" envolve, principalmente, a composição mineralógica. De acordo com Chiodi Filho (2004), a qualidade de uma rocha é verificada pela presença mínima de minerais que sejam facilmente intemperizados no ambiente, que podem comprometer o uso e durabilidade. A utilização de diferentes litotipos depende de várias características, mas, atualmente, os mármores representam $45 \%$ da produção mundial, $40 \%$ atribuídos aos granitos, $5 \%$ aos quartzitos e similares, e $5 \%$ às ardósias. A participação dos granitos elevou-se de um patamar de $15 \%$ no início da década de 50 , para $22 \%$ na década de $70,38 \%$ em meados da década de 80 , até os atuais $40 \%$, incrementando a demanda global sem restringir a utilização dos mármores (MEC-Brasil, 2007).

Embora o país tenha papel de destaque no cenário internacional de rochas ornamentais, as observações apontadas levaram este grupo de pesquisadores a formular questões que permitissem discutir o Ciclo das Rochas, um conteúdo das Geociências definido nos Parâmetros Curriculares Nacionais (PCN's), que poderia ser desenvolvido no ensino básico.

De fato, embora Geografia seja a disciplina que tradicionalmente trata de vários conteúdos das Geociências, a fragmentação dos conteúdos em tópicos em determinados momentos não contribui para que o aluno desenvolva uma noção do ciclo global, dos processos da dinâmica terrestre (Toledo, 2005). Ressalta-se, ainda, a falta de conexão entre os conteúdos e outras Ciências, como Química, Física, inerentes tanto aos processos quanto às características dos materiais geológicos.

Assim, a partir do estudo do som produzido pelas rochas encontramos possibilidades várias que permitiriam uma abordagem interdisciplinar no ensino de Geociências e de Física, além de outros conteúdos que poderão ser associados.

\section{Objetivos e Metodologia}

O objetivo principal deste trabalho é estabelecer uma proposta para a utilização do litofone como ferramenta educacional no ensino de ciências. No desenho e montagem do instrumento musical, tópicos em geologia e física são explorados. Em particular, mostramos como técnicas espectrais envolvendo o som pode ser empregadas como ferramentas para a caracterização de rochas.

No presente estudo selecionamos dois litotipos comumente utilizados como rochas ornamentais 

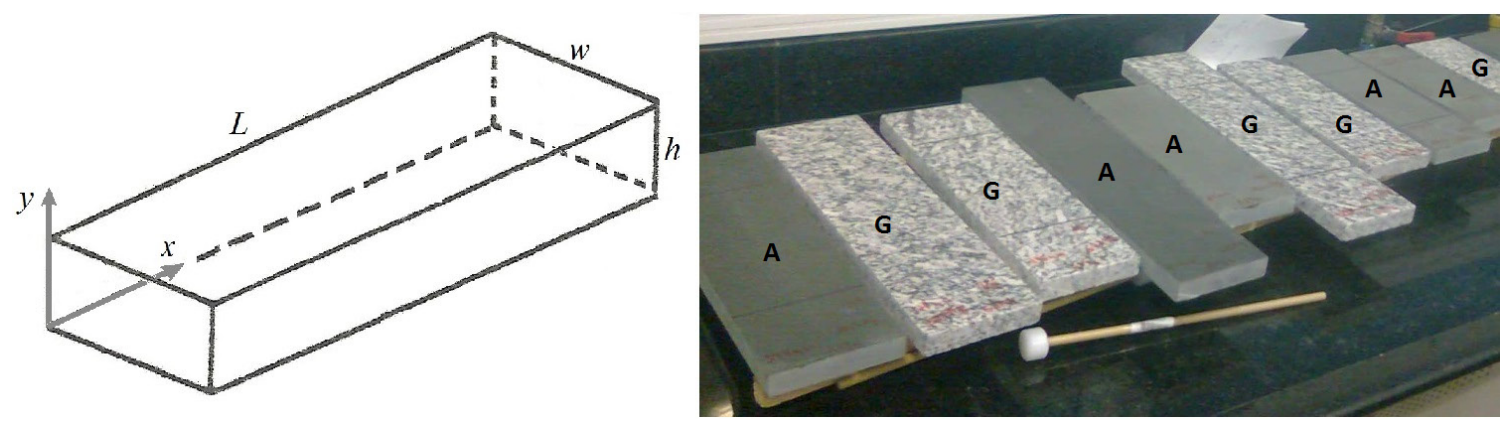

Figura 1. Esquerda: Geometria das placas de rocha. Direita: Algumas amostras da placa de granito (G) e ardósia (A) e a baqueta utilizada. A ponta da baqueta é feita de nylon e as amostras estão assentadas sob dois suportes de madeira.

no Brasil, em função das características apresentadas e o custo, a ardósia e o granito.

As amostras foram cortadas na forma de placas, todas com a mesma altura $(\mathrm{h}) \mathrm{em}$ torno de $0,022 \mathrm{~m}$ e a largura $(\mathrm{w})$ em torno de $0,103 \mathrm{~m}$. O comprimento (L) das seis placas variou entre $0,174 \mathrm{~m}$ e $0,437 \mathrm{~m}$ (ver a Figura 1 para uma descrição da geometria das placas de rocha). Os comprimentos foram definidos de forma que não fossem múltiplos um do outro. As amostras foram cortadas em duplicata, resultando doze amostras do granito e doze amostras da ardósia, com seis comprimentos diferentes.

Para o registro do som produzido utilizou-se um microfone padrão, comum em pacotes multimídia de computadores. A taxa de amostragem do som gravado foi de $8 \mathrm{kHz}$ a $42 \mathrm{kHz}$, dependendo da frequência do som. A fim de reduzir o amortecimento, as placas foram apoiadas sobre madeira, a $22,4 \%$ e $77,6 \%$ do comprimento da placa.

Para efeito de comparação entre os sons produzidos pelas placas de rocha, utilizamos, também, dois instrumentos de produção de som: um cilindro de alumínio, conhecido como "sino de meditação" ou "som interior"; e um metalofone (xilofone de metal para crianças). O diâmetro (a) da placa cilíndrica é de $0,0125 \mathrm{~m}$ e o seu comprimento é de $0,133 \mathrm{~m}$. O metalofone é qualquer instrumento musical composto de placas de metal afinadas que são atingidas com uma baqueta para fazer um som musical. A placa do metalofone utilizado tem $\mathrm{L}=$ $0,107 \mathrm{~m}, \mathrm{w}=0,028 \mathrm{~m}, \mathrm{~h}=0,002 \mathrm{~m}$, embora a forma da placa não seja exatamente retangular (Figura 2).

O sinal gravado em arquivo do tipo wave é apresentado como uma função do tempo, nas Figuras 3, 4 e 5. No lado esquerdo dessas figuras, há um destaque para o formato da onda, e no lado direito, o sinal é mostrado em um intervalo de tempo mais longo. $\mathrm{O}$ decaimento da amplitude do som é mais rápido no caso das rochas do que nos casos dos instrumentos metálicos. A forma de onda do som do cilindro de alumínio é senoidal e a forma de onda das rochas e do metalofone são mais ricos que a onda do bastão de alumínio. A forma da onda sonora depende de quais frequências estão presentes e da razão entre as amplitudes das frequências da onda sonora. A forma de onda também está relacionada com o timbre do instrumento musical.

As vibrações transversais em uma placa colocada horizontalmente (Fig. 1) obedecem à equação

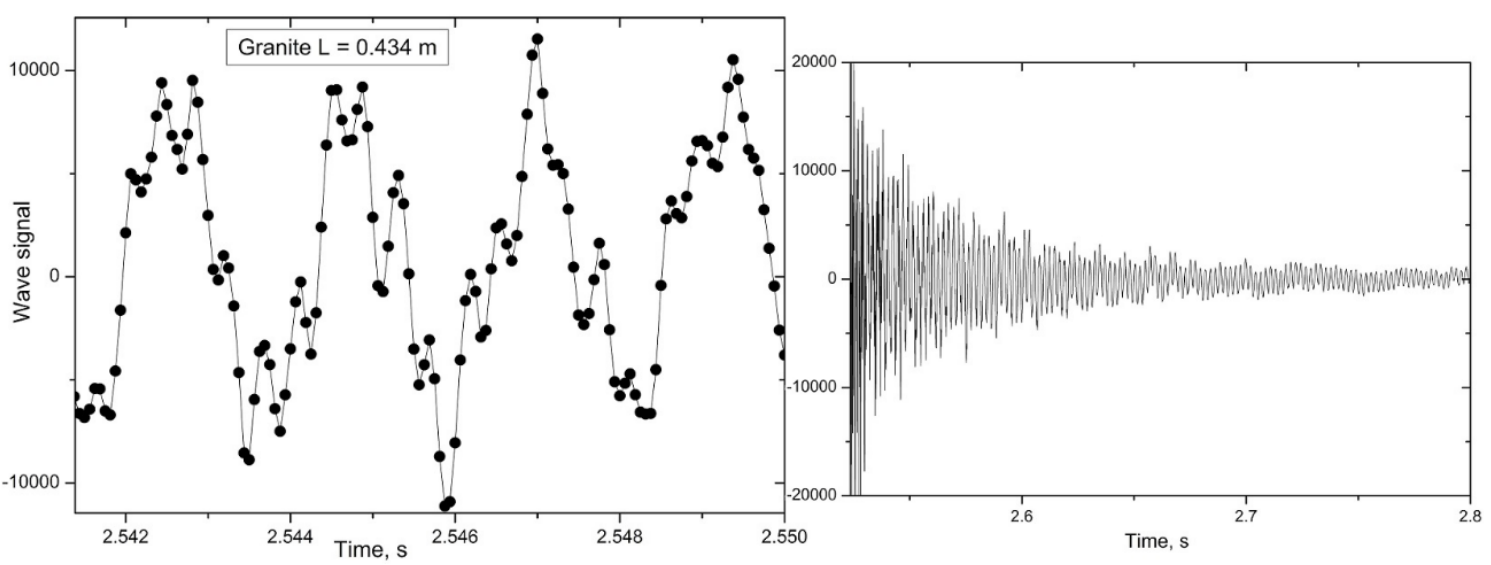

Figura 2. Esquerda: bastão de alumínio maciço usado para meditação, e sua baqueta de madeira. Direita: xilofone de metal infantil (metalofone), analisamos o som da placa mais longa. 


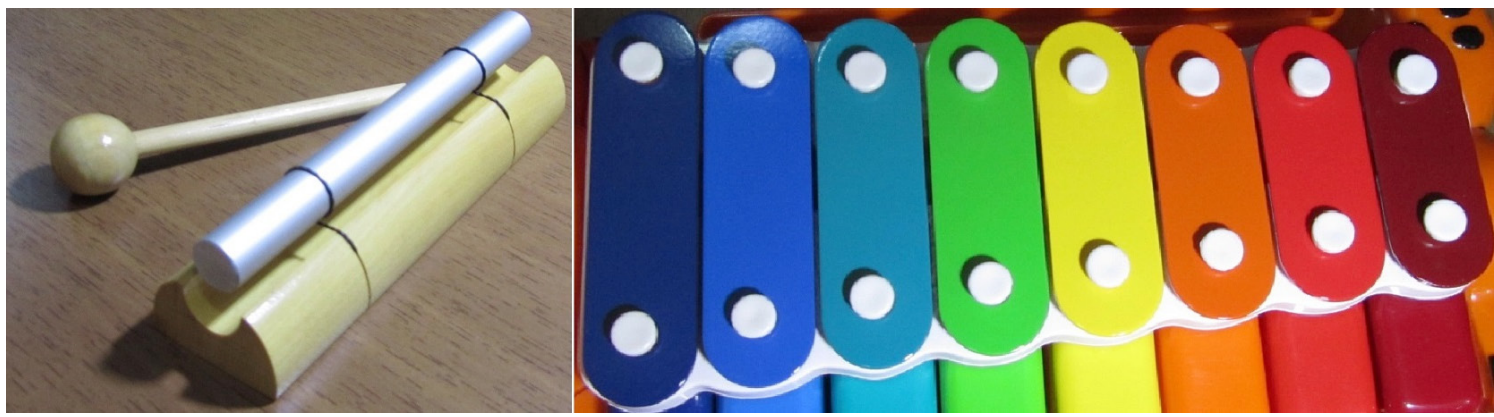

Figura 3. Sinal de onda gravado para uma placa de granito, como função do tempo, para dois intervalos de tempo diferentes.

de onda m (Fletcher et al. 1991):

$$
\frac{\partial^{2} y}{\partial t^{2}}=-\frac{K^{2}}{\rho} \frac{\partial^{4} y}{\partial x^{4}} \quad \text { Equação } 1
$$

onde $\boldsymbol{t}$ é o tempo, a direção de $\boldsymbol{y}$ é vertical e a direção de $\boldsymbol{x}$ é horizontal; $\boldsymbol{E}$ é o módulo de Young, $\boldsymbol{r}$ é a densidade do material, e $\boldsymbol{K}$ é o raio de giração da seção transversal da placa. No caso de placas retangulares $K=\boldsymbol{h} /($ Ö12), e para a placa cilíndrica (bastão) $K=\boldsymbol{a} / 4$.

Módulo de Young, $\boldsymbol{E}$, é também denominado módulo de elasticidade e mede a rigidez de um material.

As condições de contorno para placas que podem vibrar com as extremidades livres geram as frequências dadas por (Fletcher et al., 1991):

$$
f_{n}=\frac{\pi K}{8 L^{2}} \sqrt{\frac{E}{\rho}}\left[3.011^{2}, 5^{2}, 7^{2}, \ldots,(2 n+1)^{2}\right]
$$

\section{Equação 2}

Estas frequências são das oscilações naturais, ou de ressonância, do sistema. A primeira frequência também é chamada de frequência fundamental e os modos $\operatorname{com} \boldsymbol{n} 1$ são os tons mais altos.
Em 1822, Fourier demonstrou que uma função periódica pode ser decomposta em uma série de funções harmônicas simples (senos e cossenos) de amplitudes e fases adequadas. Em Santos (2013), os autores aplicaram a análise espectral de Fourier com o uso da técnica de Transformada Rápida de Fourier (FFT) para encontrar as frequências de ressonância do som. FFT é um algoritmo de computador utilizado para calcular o método de Fourier que transforma uma função do tempo em função da frequência. Os picos no perfil de amplitude ou potência são as frequências de ressonância do sistema.

\section{Análise espectral do som}

Usando o método de FFT, nós analisamos o som de doze placas de ardósia, e doze placas de granito, em comparação a um bastão de alumínio e a nota Dó do metalofone. Nós mostramos o espectro da placa de granito na Figura 6. Os espectros presentes na Figura 7 são do "sino de meditação" e do metalofone.

As proporções das primeiras três frequências destes três sistemas estão próximas aos valores previstos pela Equação 2, como mostrado na Tabela 1.

Na Figura 8, mostramos o logaritmo da primei-

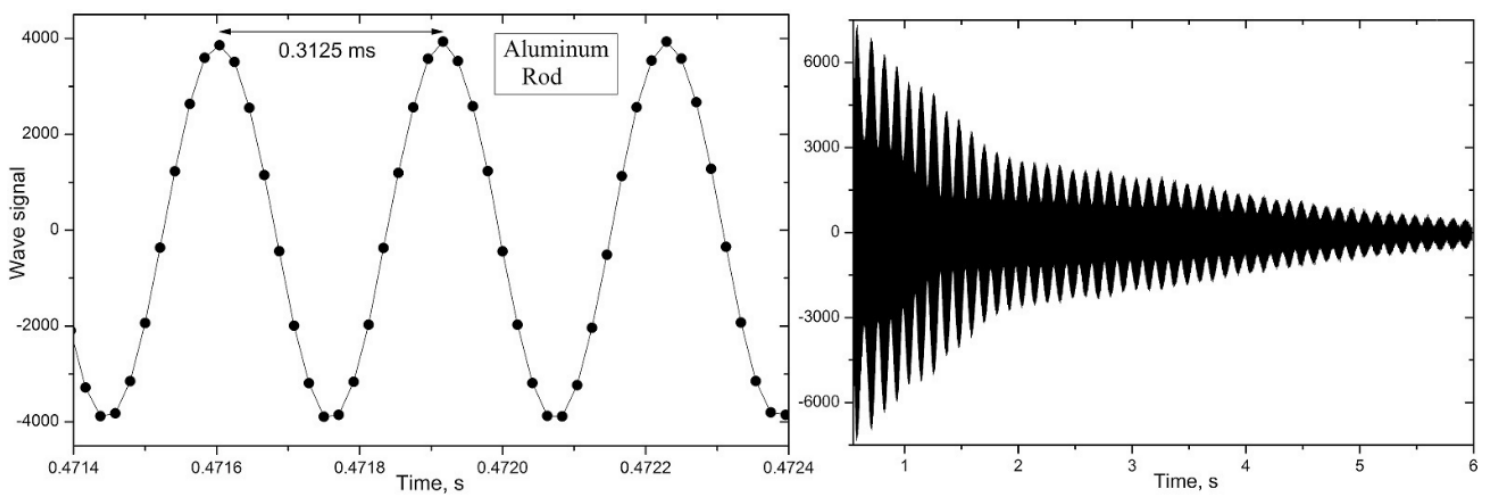

Figura 4. Sinal de onda gravado para o bastão cilíndrico de alumínio (sino de meditação) em função do tempo, para dois diferentes intervalos de tempo. A esta onda tem forma senoidal. 


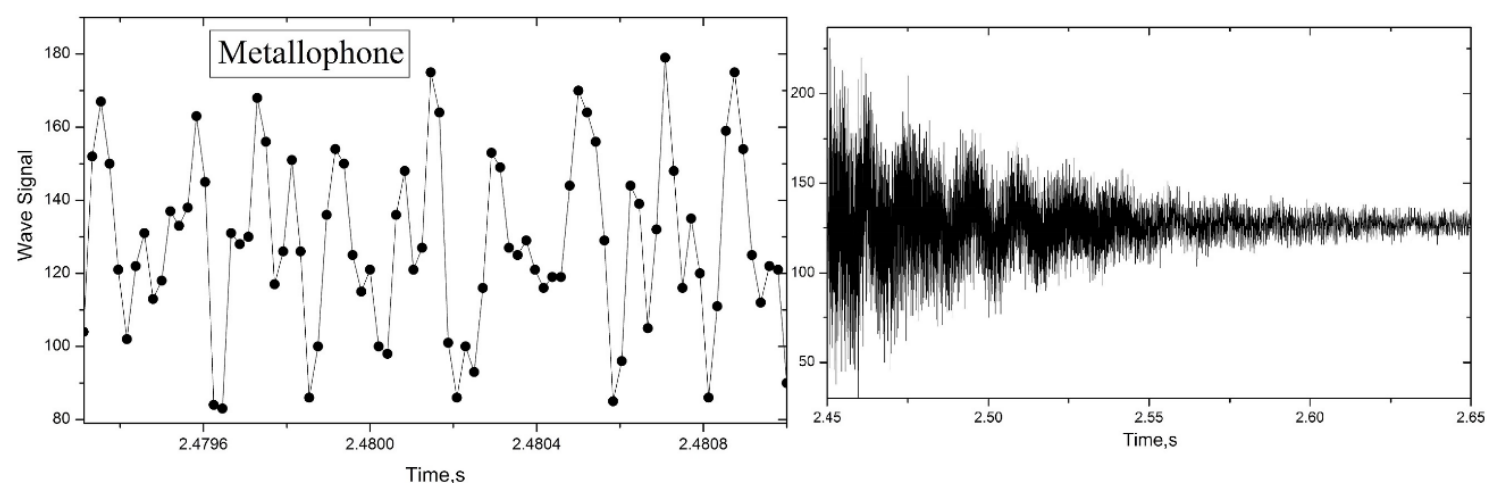

Figura 5. Sinal de onda gravado para uma placa de nota Dó do metalofone em função do tempo, para dois diferentes intervalos de tempo

ra frequência de cada placa de rocha em função do comprimento da placa. Os dados experimentais estão de acordo, como previsto na teoria (Eq. 2), isto é, a frequência é inversamente proporcional ao quadrado do comprimento.

Nós medimos comprimento, largura e altura (respectivamente $L, w, h$ ) e a massa das placas de rocha, para calcular a densidade ( $\rho=$ massa/volume) das amostras. A altura média das amostras é de $0,0211 \mathrm{~m}$ para as placas de granito e de 0,0221 $\mathrm{m}$ para as placas de ardósia. Usando os valores de $\rho, \mathrm{L}, \mathrm{h}$ e os coeficientes lineares das funções ajustadas aos dados experimentais mostradas na Figura 8, calculamos o módulo de Young $(E)$ das rochas. Os valores da densidade e do módulo de Young são apresentados na Tabela 2.

O módulo de Young é um parâmetro crítico para descrever o comportamento das rochas sobre carga (Santi, 2000). Para as placas de rocha nós usamos o coeficiente linear das funções ajustadas, para o bastão de alumínio nós usamos somente a frequência fundamental $\left(f_{1}\right)$, a densidade $(\rho)$, a altura $(L)$ e o diâmetro $(a)$ do bastão na equação 2.

Com as funções das frequências fundamen-

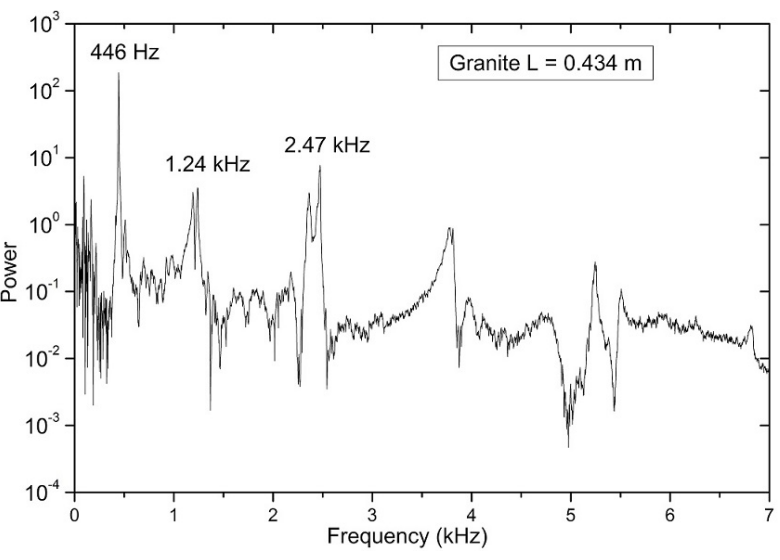

Figura 6. Espectros de potência do som da placa de granito

tais em função do comprimento das placas de rocha, podemos escolher as frequências das notas musicais que queremos e cortamos as placas de tal forma que os comprimentos correspondam às frequências musicais. Pequenos ajustes de afinação são necessários.

Neste ponto, um exemplo numérico pode ser útil. Tomando as placas de granito disponíveis, temos a partir da Tabela 2 a densidade e módulo de Young associados ao material, respectivamente
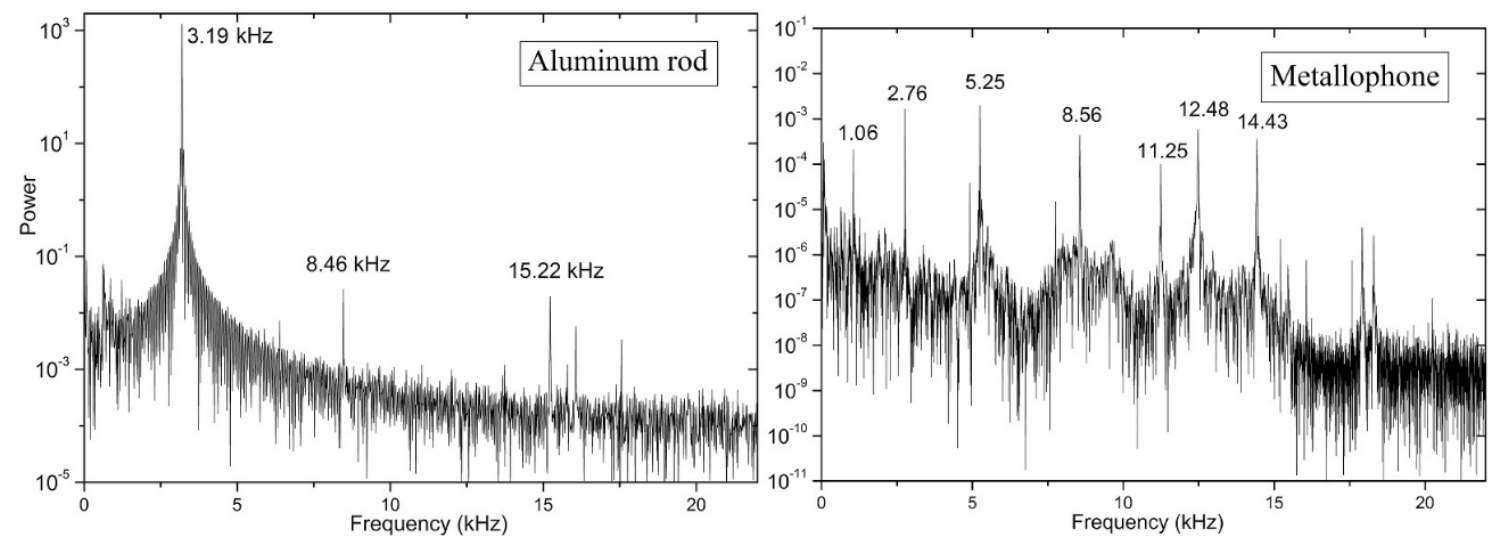

Figura 7. Espectros de potência do som do sino de meditação (esquerda) e de uma placa Dó do metalofone (xilofone de metal de uma criança) (direita) 


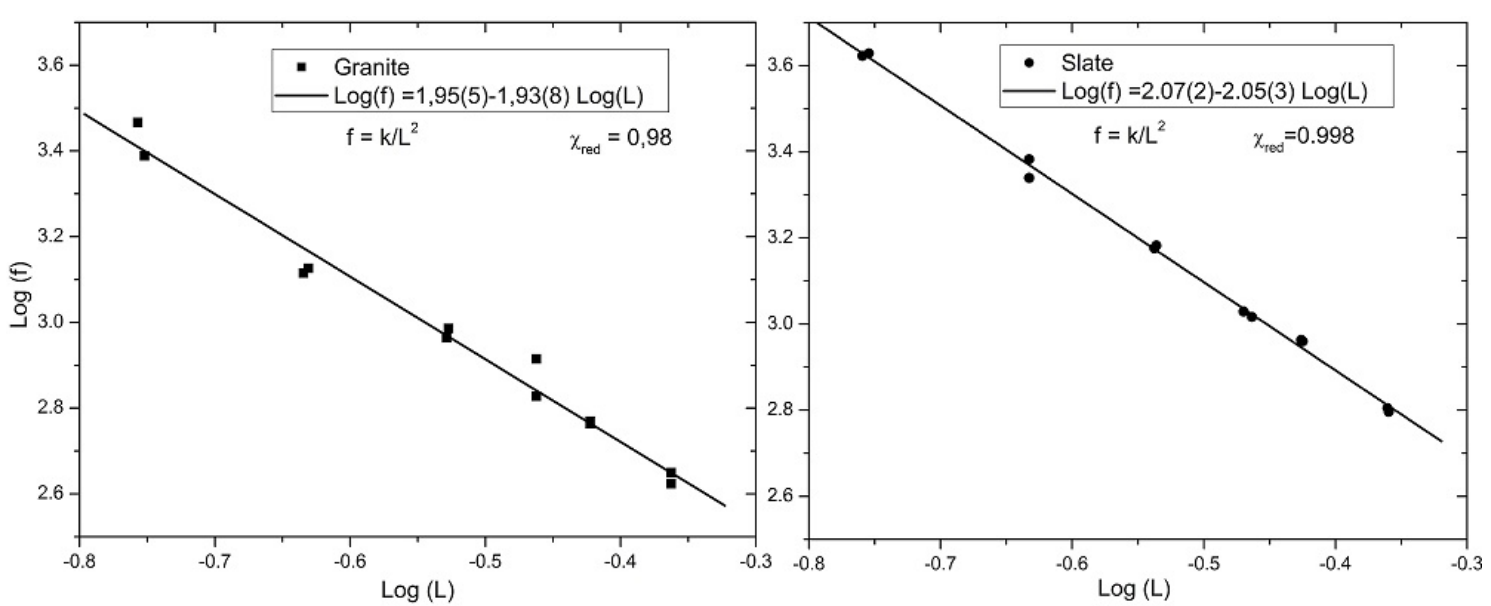

Figura 8. A frequência fundamental (f1) em função do comprimento (L) das placas de rocha em escalas logarítmicas. A função descrita pela Equação 2 é ajustada aos dados

$2,85 \times 10^{3} \mathrm{~kg} / \mathrm{m}^{3}$ e $48 \mathrm{GPa}\left(48 \times 10^{9} \mathrm{~Pa}\right)$. Por intermédio da Eq. 1, vemos que, se cortarmos uma placa de granito de $0,434 \mathrm{~m}$, teremos uma frequência fundamental próxima de $446 \mathrm{~Hz}$, experimentalmente observada (Fig. 6).

\section{Conclusões}

A partir do uso de um computador pessoal com um microfone comum e software para realizar a análise espectral, o som de instrumentos musicais feitos de placas de rocha pode ser estudado. A análise dos dados observados, conforme previstos por um modelo físico simples, permite a construção de um litofone.

A abordagem apresentada possibilita a caracterização mecânica de materiais, e desta forma, quando efetuamos a comparação entre os dados obtidos para diferentes litotipos, podemos estabelecer discussão sobre a composição mineralógica, estruturas, foliações, entre outras propriedades mecânicas das rochas.

O apelo da música e instrumentos musicais podem transformar um projeto como a construção de um litofone em uma ferramenta educacional entre o ensino de Geologia, e outras áreas, como Física e Artes, por exemplo, de forma interdisciplinar. $O$ trabalho apresentado pode ser útil até mesmo em áreas aparentemente não relacionados, tais como Fisiologia, uma vez que os nossos ouvidos realizam uma espécie de análise de frequência.

\section{Referências}

Bastos P.L. 2010. Small holes of wonder. In: Second Vienna Talk, Sept. 19-21, 2010. Proc..., Univ. Music and Performing Arts Vienna, Austria.

Chiodi Filho C., Rodrigues E.P., Artur A.C. 2004. Panorama Técnico - Econômico do Setor de Rochas Ornamentais no Brasil. São Paulo, Revista Geociências, 23(1/2):5-20.

Fletcher N.H., Thomas D.R. 1991. The Physics of Musical Instruments. New York, Springer.

Kochkin N. I., Chirkevitch M.G. 1986. Prontuário de Física Elementar. Moscow, Mir.

Brasil. Ministério da Educação (MEC). 2007. Rochas Ornamentais. Ministério da Educação/Secretaria de Educação Profissional e Tecnológica. Brasília, DF. 29p.

$\mathrm{Ng}$ K., Ong B. 2010. Interactive Multimedia Rocks for Geology. In: Intern. Conf. Electronic Visualisation and the Arts, British Computer Society, 2010.

Santi P.M., Holschen J.E., Stephenson R.W. 2000. Improving Elastic Modulus Measurements for Rock Based Geology. Environ. \& Eng. Geoscience, VI(4):333.

Santos E.M., Molina C., Tufaile A.P.B. 2013. Violão e guitarra como ferramentas para o ensino de física. Rev. Bras. Ens. Física, 35(2).

Toledo M.C.M. 2005. Geociências no Ensino Médio Brasileiro. Análise dos Parâmetros Curriculares Nacionais. São Paulo, Rev. Geologia USP. Publ. Esp., 3:33-34. 